MS2-05 The newPin project: Towards a new sample holder standard for cryogenic macromolecular x-ray crystallography

Gergely Papp ${ }^{1}$, Christopher Rossi ${ }^{1}$, Clement Sorez ${ }^{1}$, Franck Felisaz $^{1}$, Hassan Belrhali ${ }^{1}$, Matthew Bowler ${ }^{1}$, Florent Cipriani ${ }^{1}$

1. European Molecular Biology Laboratory, Grenoble Outstation, France

email: gpapp@embl.fr

The SPINE sample holder standard [1] has been essential for the automation of macromolecular X-ray crystallography (MX) in Europe. Motivated by the continuously increasing sample flow at MX beamlines and by the emergence of automated harvesters, studies are ongoing to define a compact and precise sample holder for frozen crystallography. Lead by the EMBL Grenoble, this "newPin" collaborative project is partly supported by the European FP7 program BioStruct-X (www.embl.fr/newpin) and includes most of the European synchrotrons, NSLS-II (USA), SPING8 (Japan), as well as the companies Molecular Dimension Ltd. and MiTeGen. First aim of this new standard is to reduce the transporting costs of frozen samples as well as puck turnover at beamlines. Second aim would be to facilitate crystal alignment at beamlines using a design that allows precise 3D positioning of the sample holder on its support. Ultimately, automated harvesting systems like "CrystalDirect" [2] could record the coordinates of the harvested crystals for a priori alignment at beamlines. Two sample holder models are proposed with a storage density of 36 samples per puck, newPin and miniSpine, which share a common handling robot gripper and uni-puck like Dewar slots. MiniSpine was selected for the first implementation of the future sample holder standard as it is easier to integrate at beamlines. It will be presented in detail together with compatible robotics and goniometer equipment. The results obtained at the ESRF/EMBL/INDIA BM14 beamline using the FlexED8 sample changer will be shown. The challenges to finalize and integrate this new standard at beamlines will be discussed.

[1] Acta Cryst. (2006) D62, 1251-1259

[2] Acta Cryst. (2012). D68, 1393-1399
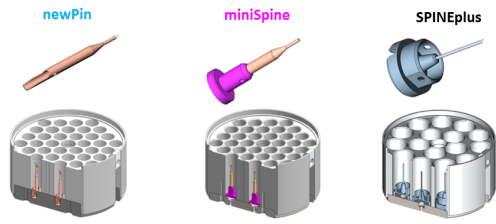

Figure 1. newPin, miniSpine and SPINEplus sample holders and pucks

Keywords: sample holder, cryogenic sample holder, SPINE standard, sample changer, MX, macromolecular crystallography
MS3 Data collection and

processing software (XFELS \&

synchrotrons)

Chairs: Adrian Mancuso, Thomas Schneider

MS3-01 MX data analysis developments at Diamond Light Source.

Gwyndaf Evans ${ }^{1}$, Melanie Vollmar ${ }^{1}$, Graeme Winter ${ }^{1}$, David Waterman ${ }^{2}$, James Parkhurst ${ }^{1}$, Richard Gildea ${ }^{1}$, Pierre Aller ${ }^{1}$, James Foadi ${ }^{1}$, Alun Ashton ${ }^{1}$

1. Diamond Light Source, Harwell Science and Innovation Campus, Didcot OX11 0DE, United Kingdom.

2. CCP4, Research Complex at Harwell, Rutherford Appleton Laboratory, Didcot OX11 0FA, UK

email: gwyndaf.evans@diamond.ac.uk

The Macromolecular Crystallography team at Diamond Light Source are providing their users with a broad suite of beamlines and capabilities all supported by automated data analysis engines running in the background, generating results that are accessible to remote users through web and app interfaces. The new VMX beamlines at Diamond and the Diamond XFEL-Hub will push the limits of perfomance of these pipelines and are demanding fundamental development of underlying software and algorithms, in particular raw data analysis, to handle broad bandpass beams and multicrystal analysis. The DIALS software is one such development that provides the flexibility to accommodate these challenges and together with new approaches to pipeline design and remote analysis services (in collaboration with CCP4) we aim to provide users with 'cloud' like access to collection and analysis.

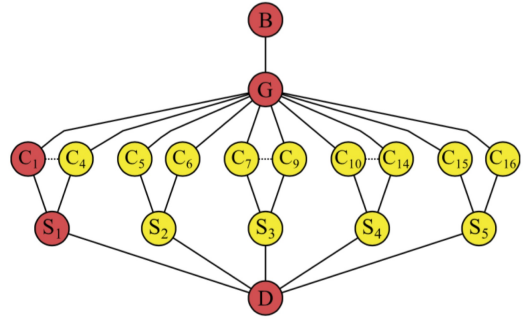

Figure 1. Global refinement model in DIALS enabling joint refinement of multi-scan multicrystal data sets or serial snapshot crystallography data. A single experiment consists of a path from top to bottom connecting one of each type of model. Key: B: beam, G: goniometer, C: crystal, S: scan and D: detector. 
Keywords: data collection, data analysis, macromolecular crystallography, DIALS, automation, pipelines

\section{MS3-O2 Hidden treasure in serial femtosecond diffraction}

Anton Barty ${ }^{1}$

\section{CFEL/DESY}

email: anton.barty@desy.de

Serial Femtosecond Crystallography using X-ray free electron lasers has established itself as a technique ideally suited to the study small and radiation sensitive crystals by outrunning radiation damage, and for probing protein dynamics with sub-picosecond time resolution. A typical experiment involves exposing a stream of crystals to millions of individual femtosecond duration X-ray pulses, resulting in measurable diffraction patterns from thousands of individual protein micro- or nano-crystals each of which is exposed to the intense X-ray beam only once before being discarded. Combining these diffraction patterns yields intensity measurements for structure analysis, for example reflection intensities for conventional crystallographic analysis. In some cases it can also reveal hidden gems including diffraction beyond the resolution of observable Bragg peaks and continuous diffraction from individual asymmetric units. This has required the development of new data analysis techniques.

Keywords: serial femtosecond crystallography, Free electron laser, data processing 